\title{
Manejo cirúrgico de fratura de mandíbula: relato de caso
}

\author{
Surgical management of mandibular fracture: case report
}

\author{
Rafael Drummond Rodrigues* \\ Paloma Heine Quintas** \\ Lucas da Silva Barreto ${ }^{* * *}$ \\ Cesar Feitoza Bassi Costa ${ }^{* * *}$ \\ Larissa Oliveira Ramos Silva**** \\ Jeferson Freitas Aguiar**
}

\section{Resumo}

Objetivo: descrever, por meio de um relato de caso, uma fratura de mandíbula em região de parassínfise esquerda, causada com agressão física. Relato de caso: paciente do sexo feminino, 26 anos de idade, ASA I, melanoderma, vítima de agressão física, procurou o serviço de Cirurgia e Traumatologia Bucomaxilofacial da Faculdade de Odontologia da Universidade Federal da Bahia. Ao exame físico, referiu queixas álgicas espontâneas em região mandibular esquerda e presença de degrau ósseo em região passinfisária esquerda. Ao exame de imagem, observaram-se sinais sugestivos de fratura em região de parassínfise esquerda. Na paciente em questão, foi realizado o manejo cirúrgico da fratura com auxílio de miniplacas e parafusos do sistema $2 \mathrm{~mm}$ em acesso intraoral. No acompanhamento pós-operatório, observaram-se material de osteossíntese e odontossíntese em posição, fratura adequadamente reduzida e ausência de sinais de deslocamento dos cotos fraturados. Considerações finais: o uso do sistema de fixação interna rígida é indicado em casos de fraturas desfavoráveis de mandíbula, uma vez que apresenta resultados satisfatórios referentes a estabilização e cicatrização óssea. A redução e a fixação com auxílio de miniplacas e parafusos tendem a evitar o deslocamento dos cotos fraturados e a formação de pseudoartrose.

Palavras-chave: Fixação interna de fraturas. Mandíbula. Traumatismos faciais.

\section{Introdução}

O trauma é consequência de uma força capaz de gerar qualquer alteração funcional e anatômica em uma determinada região do corpo humano. ${ }^{1} \mathrm{O}$ trauma de face, também denominado trauma bucomaxilofacial, corresponde a todo e qualquer ferimento físico que comprometa as estruturas anatômicas nos terços superior, médio e inferior da face. ${ }^{2}$ Tais ferimentos são capazes de acarretar lesões em estruturas ósseas bem como em tecido mole facial.

A face é considerada uma das regiões mais vascularizadas e enervadas do corpo, sendo facilmente acometida por infecções, devido ao seu rico suprimento sanguíneo. ${ }^{2}$ Sendo assim, a abordagem terapêutica das lesões bucomaxilofaciais contribui para menor morbidade do paciente, diminuindo as complicações inerentes à lesão tecidual, como infecções e consolidação desfavorável de lesões ósseas. ${ }^{3}$

A mandíbula é considerada a segunda região mais prevalente nos traumas de face, ficando atrás apenas das fraturas nasais. ${ }^{4}$ A mandíbula está envolvida entre $36 \%$ e $70 \%$ dos casos de fratura bucomaxilofacial. ${ }^{3}$ Entre as regiões mais acometidas nesses ossos, estão as regiões de côndilo e parassínfise mandibular que, muitas vezes, estão associadas ao mesmo trauma. ${ }^{2}$

A etiologia das fraturas mandibulares pode variar, sendo acidentes de trânsito, agressões físicas, quedas, acidentes desportivos e lesões por projétil Brasil.

Residente de Cirurgia e Traumatologia Bucomaxilofacial, Universidade Federal da Bahia e Hospital Santo Antônio (Obras Sociais Irmã Dulce), Salvador, BA, Brasil.

Residente de Cirurgia e Traumatologia Bucomaxilofacial, Universidade Federal da Bahia e Hospital Santo Antônio (Obras Sociais Irmã Dulce), Salvador, BA, Brasil.

***** Residente de Cirurgia e Traumatologia Bucomaxilofacial, Universidade Federal da Bahia e Hospital Santo Antônio (Obras Sociais Irmã Dulce), Salvador, BA, Brasil.

****** Preceptor do Serviço de Cirurgia e Traumatologia Bucomaxilofacial da Universidade Federal da Bahia e Hospital Santo Antônio (Obras Sociais Irmã Dulce), Salvador, Bahia, Brasil. 
de arma de fogo os mais prevalentes, respectivamente. ${ }^{5,6}$

A mandíbula é considerada um osso ímpar, localizada no terço inferior da face, articula-se com a cavidade glenoide do osso temporal, em que forma a articulação temporomandibular (ATM). ${ }^{7}$ Nesse osso, inserem-se músculos responsáveis por deglutição, fonação, mastigação e expressão facial. ${ }^{7,8}$ Por isso, quando fraturada, os cotos mandibulares fragmentados podem deslocar-se, impedindo a cicatrização óssea adequada.

Os sinais e sintomas mais associados às fraturas de mandíbula são limitação da abertura bucal, edema, assimetria facial, maloclusão, mobilidade atípica à manipulação, crepitação, parestesia e dor., ${ }^{4,5,9}$

Apesar de o exame clínico ainda ser considerado soberano no diagnóstico de fratura de mandíbula, os exames de imagem são comumente utilizados, no intuito de complementar o diagnóstico e haver maior acurácia da localização e da extensão da fratura. ${ }^{4,10}$ Entre as radiografias bidimensionais mais utilizadas, destacam-se a radiografia panorâmica e a póstero-anterior de face ou mandíbula (PA). Em alguns casos, pode-se lançar mão de outras tomadas radiográficas, como a towne reversa, lateral oblíqua de mandíbula e ATM.5,11,12

A tomografia computadorizada (TC) é também largamente utilizada, por ser considerada um exame de imagem tridimensional capaz de detalhar mais a fundo a extensão da lesão, uma vez que seus diferentes cortes tomográficos reduzem a sobreposição de estruturas anatômicas..$^{13}$ Assim, estruturas nobres adjacentes são facilmente visualizadas, 0 que contribui para um planejamento pré-cirúrgico melhor. ${ }^{4,13}$

As abordagens terapêuticas consistem em conservadoras ou cirúrgicas. ${ }^{12}$ A primeira consiste, na maioria dos casos, na estabilização da fratura por um bloqueio maxilomandibular, por um período de tempo pré-estabelecido, juntamente com terapia medicamentosa, fisioterapia e dieta líquida/pastosa rigorosa. ${ }^{7,14}$ Geralmente, esse tipo de abordagem é mais indicado em fraturas de côndilo mandibular. ${ }^{7}$

O manejo cirúrgico dessas fraturas consiste, basicamente, na redução e na fixação interna rígida dos cotos fraturados com auxílio de miniplacas e parafusos. ${ }^{1}$ Esse tratamento é largamente indicado em casos de fraturas complexas e desfavoráveis da mandíbula. ${ }^{7} \mathrm{~A}$ fixação desses cotos busca reduzir a mobilidade da fratura, impedindo a formação de tecido de interposição entre os ossos que dificulte sua cicatrização, quadro denominado pseudoartrose. ${ }^{15}$

O objetivo deste trabalho consiste em descrever, por meio de um relato de caso, uma fratura de mandíbula em região de parassínfise esquerda, causada com agressão física.

\section{Relato de caso}

O presente trabalho foi submetido ao Comitê de Ética em Pesquisa da Faculdade de Odontologia da Universidade Federal da Bahia sob o CAAE no 96586818.3.0000.5024.

Paciente do sexo feminino, 26 anos de idade, ASA I, melanoderma, vítima de agressão física no dia 22 de abril de 2018, procurou o serviço de Cirurgia e Traumatologia Bucomaxilofacial da Faculdade de Odontologia da Universidade Federal da Bahia.

Como queixa principal, a paciente relatou "precisar fazer cirurgia, pois partiu o queixo", após trauma contuso em região mentual. A paciente negou uso crônico de medicamentos, alergias medicamentosas e patologia de base. Referiu tabagismo e etilismo.

$\mathrm{Na}$ anamnese, a paciente referiu queixas álgicas espontâneas em região mandibular esquerda. Ao exame físico bucomaxilofacial, foram constatadas higiene oral regular, abertura bucal medindo $25 \mathrm{~mm}$, odontossíntese com fio de aço em posição nos elementos 31, 32 e 33. Ainda, notaram-se fratura coronária da unidade 25 , presença de degrau ósseo em região passinfisária esquerda, côndilos preservados e funcionais e edema discreto em região mandibular do lado esquerdo (Figura 1 e 2).

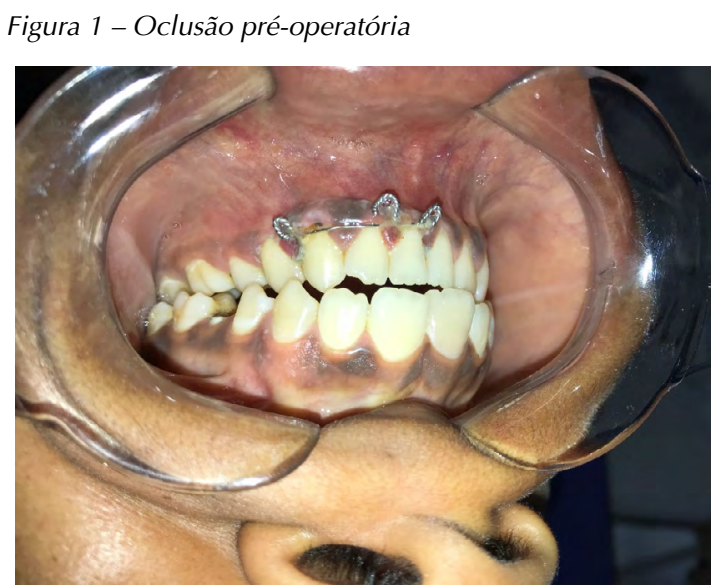

Fonte: autores.

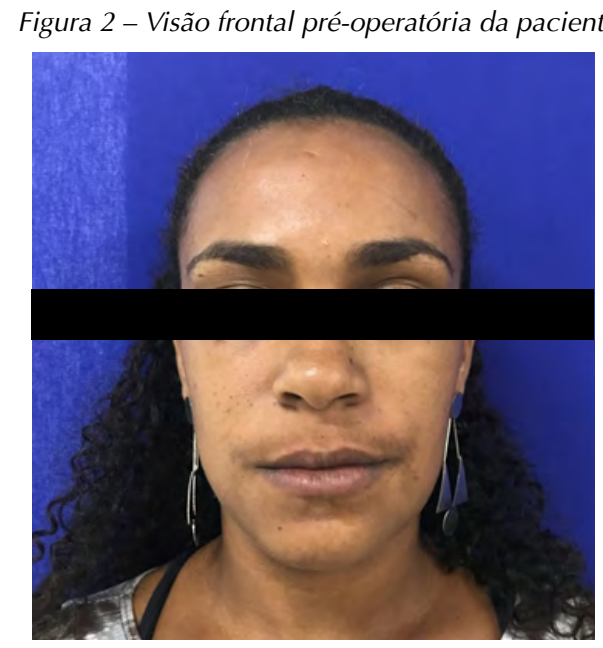

Fonte: autores. 
Ao exame de imagem, observaram-se sinais sugestivos de fratura em região de parassínfise mandibular esquerda (Figura 3).

Figura 3 - Reconstrução em 3D (TC) evidenciando traço de fratura

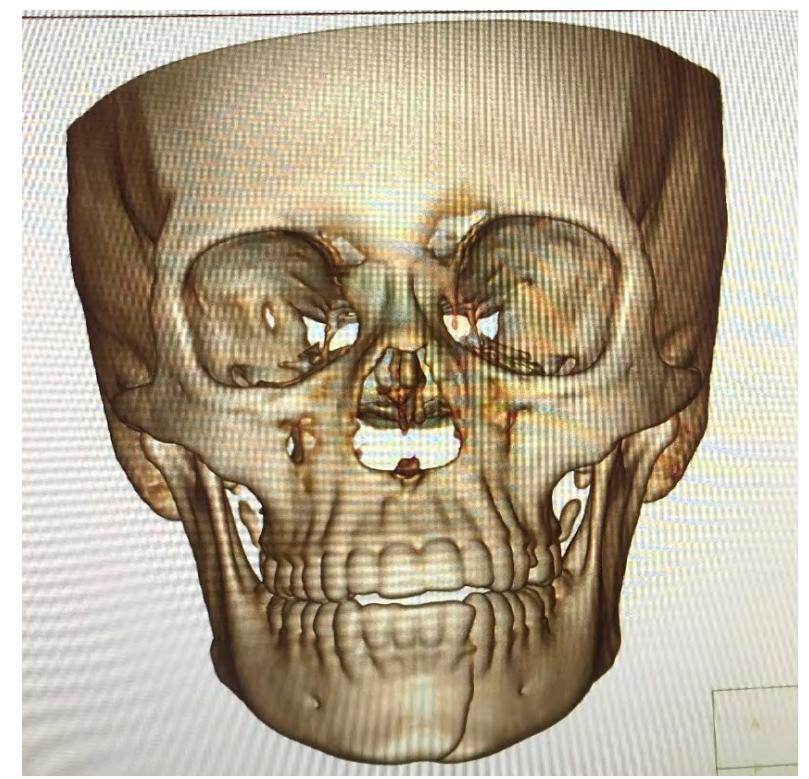

Fonte: autores.

Após as avaliações clínica e imaginológica da fratura, foi planejada a abordagem cirúrgica para a redução e a fixação interna rígida com miniplacas e parafusos do sistema $2 \mathrm{~mm}$.

Feitos os exames laboratoriais pré-operatórios, não havendo alterações significativas, a cirurgia foi realizada no dia 08 de maio de 2018, no Hospital Manoel Victorino, localizado em Salvador, Bahia.

Com a paciente em posição de decúbito dorsal, com intubação nasotraqueal e sob anestesia geral, foram realizadas a antissepsia com digluconato de clorexidina e a aposição dos campos operatórios. Ainda, instalou-se o tampão orofaríngeo, e foi infiltrado anestésico local intraoral em região de fundo de vestíbulo esquerdo com lidocaína $2 \%$ com epinefrina 1:200.000.

Optou-se por acesso intraoral para mandíbula, com divulsão dos planos, descolamento do periósteo, exposição dos cotos fraturados e redução e fixação da fratura com duas placas do sistema $2 \mathrm{~mm}$. Foi realizada a fixação dos fragmentos em região de tensão com miniplaca de 5 furos e 4 parafusos e em região de compressão com miniplaca de 7 furos e 6 parafusos (Figura 4).
Figura 4 - Fixação interna rígida da fratura com miniplacas do sistema $2 \mathrm{~mm}$

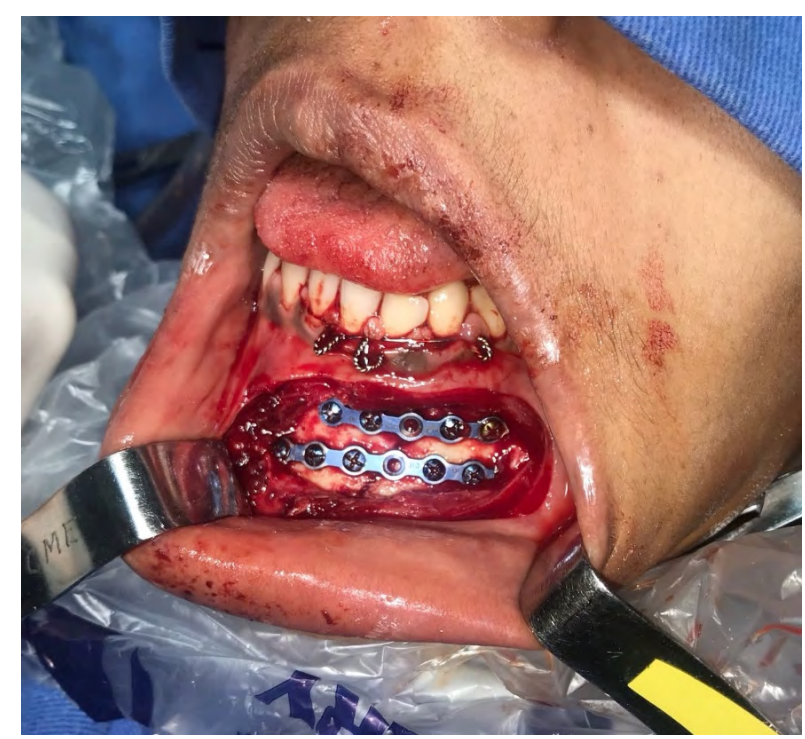

Fonte: autores.

A síntese do acesso cirúrgico foi realizada por planos, com fio de sutura reabsorvível Vycril 3-0 e 4-0. Finalmente, foram removidos o tampão orofaríngeo, os campos operatórios e a extubação nasotraqueal sem nenhuma intercorrência.

O pós-operatório a paciente cursou sem queixas álgicas, com sutura intraoral, odontossíntese em posição, sem sinais flogísticos de inflamação, edema compatível com pós-operatório, oclusão estável, abertura bucal preservada, higiene oral regular e parestesia em região mentual esquerda, que regrediu após 3 semanas da cirurgia (Figura 5).

Figura 5 - Aspecto clínico da região abordada evidenciando odontossíntese

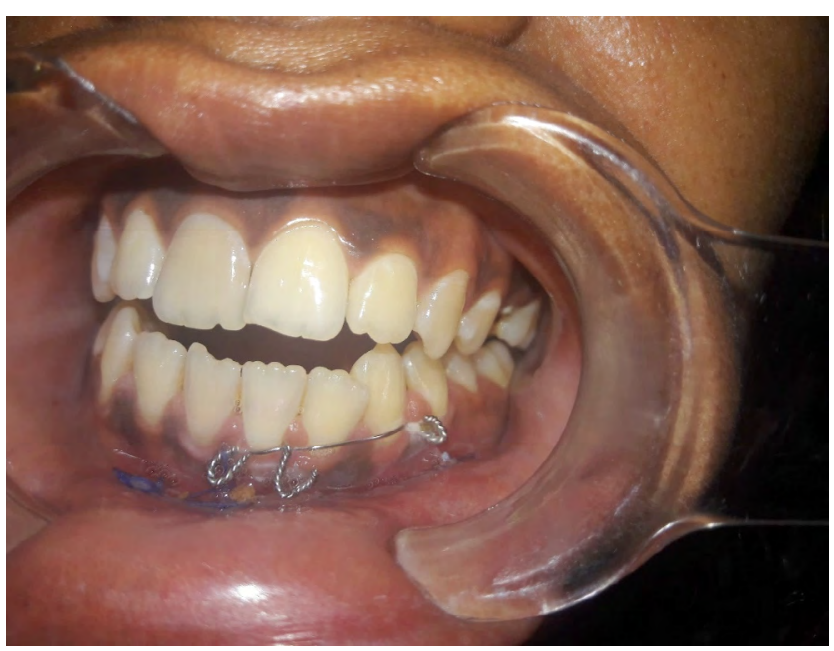

Fonte: autores.

Ao exame de imagem imediato (radiografia panorâmica), observaram-se materiais de osteossíntese e odontossíntese em posição, fratura adequadamente reduzida e ausência de sinais de deslocamento dos cotos fraturados (Figura 6). 
Figura 6 - Radiografia panorâmica pós-operatória

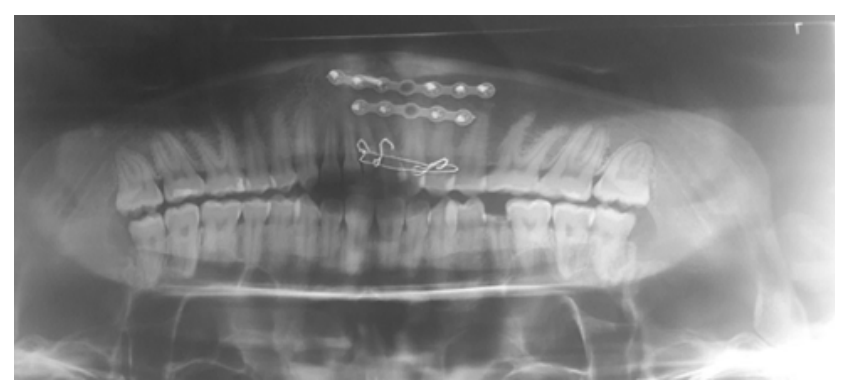

Fonte: autores.

Vale ressaltar que a paciente aceitou a publicação deste relato de caso mediante assinatura de um termo de consentimento livre e esclarecido.

\section{Discussão}

As fraturas de mandíbula têm como principal agente etiológico os acidentes de trânsito. ${ }^{7}$ Em seguida, as agressões físicas ocupam a segunda posição, como no caso relatado. Ainda, as regiões de parassínfise e ângulo mandibular são as áreas mais acometidas em fraturas complexas desse osso. ${ }^{8}$

As avaliações clínica e imaginológica são consideradas o principal método de diagnóstico em casos de fraturas de mandíbula. ${ }^{9,15}$ Clinicamente, alguns sinais e sintomas podem ser classicamente observados. Na paciente em questão, observaram-se presença de degrau ósseo, mobilidade óssea atípica à manipulação, edema local e queixas álgicas espontâneas em região mentual.

Ao exame de imagem, pôde-se observar com maior acurácia a linha de fratura e a separação dos cotos fraturados em região de parassínfise mandibular esquerda, o que corrobora com a maior prevalência de acometimento encontrado na literatura. ${ }^{8}$

Por tratar-se de uma fratura complexa com deslocamento considerável, a abordagem cirúrgica foi indicada neste caso. ${ }^{7,8} \mathrm{O}$ tratamento cirúrgico das fraturas em face objetiva reestabelecimento da função, estética e anatomia da região com auxílio de miniplacas e parafusos metálicos para esse fim. ${ }^{7,11,15}$ A adequada cicatrização ocorre graças à reaproximação dos cotos e à sua imobilidade, estabelecida pelo sistema de fixação interna rígida. ${ }^{11}$

Quando se refere aos sistemas de fixação interna rígida em cirurgia e traumatologia bucomaxilofacial, dois dispositivos podem ser utilizados. Os dispositivos load-sharing consistem em miniplacas e parafusos do sistema $1,5 \mathrm{~mm}$ a $2 \mathrm{~mm}$, que buscam o compartilhamento das cargas geradas sobre o osso fraturado. São mais indicados em casos de fraturas lineares e bem definidas e cotos fraturados com tamanhos favoráveis a redução e fixação. ${ }^{7}$

Os dispositivos load-bearing são miniplacas e parafusos do sistema $2,4 \mathrm{~mm}$ a $3 \mathrm{~mm}$, que absorvem toda a carga gerada sobre o osso, no intuito de evitar maior deslocamento e instabilidade da fra- tura. ${ }^{7}$ São majoritariamente indicados em casos de fraturas cominutivas ou perda considerável de parte óssea mandibular. ${ }^{6}$

Neste trabalho, a paciente apresentou fratura bem alinhada, sem cominução e perda de estrutura óssea que justificassem a utilização de sistema de fixação interna rígida load-bearing. Sendo assim, foram planejadas a redução e a fixação com miniplacas do sistema $2 \mathrm{~mm}$ load-sharing. Placas em região de tensão e compressão foram fixadas, no intuito de garantir maior estabilidade da fratura e cicatrização óssea mais eficiente.

O manejo tardio das fraturas de mandíbula e as suas inadequadas redução e fixação são apontados na literatura como um dos motivos que levam ao prolongamento do tempo de cicatrização óssea e a maiores chances de insucesso pós-operatório. ${ }^{1,4} \mathrm{De}$ acordo com De Paula et al. ${ }^{16}$ (2017), quando abordadas em um curto período de tempo após o trauma, as fraturas tendem a ser consolidadas em poucos meses. Entretanto, uma falha na redução dos cotos pode levar à formação de um tecido fibrótico na linha de fratura que dificulta a formação óssea e causa dor local, quadro denominado pseudoartrose. Nesses casos, uma reabordagem cirúrgica pode ser necessária. ${ }^{8,16}$

A dieta líquida e pastosa é indicada a pacientes depois do tratamento cirúrgico de fratura mandibular. ${ }^{15}$ Após fixação e redução da fratura, não é necessária a realização de odontossíntese, uma vez que tal manobra é indicada em tratamentos conservadores, em que não há necessidade de abordagem cirúrgica da fratura. ${ }^{4,7}$ Entretanto, a paciente deste estudo apresentou-se pouco colaborativa às orientações pós-operatórias, e, no intuito de garantir maior estabilidade óssea, optou-se por manter a odontossíntese em posição.

\section{Conclusão}

A abordagem cirúrgica das fraturas de mandíbula é indicada em casos em que há deslocamento considerável dos cotos fraturados. A utilização do sistema de fixação interna rígida por dispositivos load-sharing tem demonstrado eficiência na estabilização e na consolidação da fratura, sendo indicado em casos de fratura bem definida e sem perda de continuidade óssea.

\section{Abstract}

Objective: to describe, through a case report, a mandible fracture in the left paraphysis region caused by physical aggression. Case report: female patient, 26 years old, ASA I, melanomedma, victim of physical aggression, researched at the Department of Oral and Maxillofacial Surgery and Traumatology from the Federal University of Bahia. In the physical exam, the patient referred spontaneos pain in the left parasymphysis and bone stepping in passinfisary left region. At the imaging examination observed suggestive signs of a fracture in the region of the left parasymphysis. The patient in 
question was approached by a surgical procedure of the fracture with miniplates and screws of the $2.0 \mathrm{~mm}$ system in an intraoral approach. Postoperatively, osteosynthesis and odontosynthesis material was observed in position, adequately reduced fracture and absence of signs of displacement of fractured stumps. Final considerations: the use of the internal fixation system is indicated in cases of unfavorable mandibular fractures once it presents satisfactory results regarding bone stabilization and healing. The reduction and fixation with miniplates and screws tend to avoid the displacement of fractured stumps and formation of pseudoarthrosis.

Keywords: Fracture fixation. Mandible. Facial injuries.

\section{Referências}

1. Junior RC, Moraes RB, Landes C, Luz JGC. Comparison of a 2.0-mm locking system with conventional 2.0- and 2.4$\mathrm{mm}$ systems in the treatment of mandibular fractures: a randomized controlled trial. Oral Maxillofacial Surg 2017; 21(3):327-34.

2. Imai T, Sukegawa S, Kanno T, Yamamoto N, Furuki Y, Michizawa M. Mandibular fracture patterns consistent with posterior maxillary fractures involving the posterior maxillary sinus, pterygoid plate or both: CT characteristics. Dentomaxillofac Radiol 2014; 43(2):2-7.

3. Boffano P, Kommers SC, Roccia F, Forouzanfar T. Mandibular trauma treatment: a comparison of two protocols. Med Oral Patol Oral Cir Bucal 2015; 20(2):218-23.

4. Zarpellon AT, Cruz GO e A, Gus IO, Moreira GHG, Chamma RS, de Masi FDJ, et al. Review of 150 cases of mandible fracture between 2010 and 2013 at the Cajuru University Hospital, Curitiba, PR. Rev Bras Cir Plást 2015; 30(4):609-14.

5. Gadicherla S, Sasikumar P, Gill SS, Bhagania M, Kamath AT, Pentapati KC, et al. Mandibular Fractures and Associated Factors at a Tertiary Care Hospital. Arch Trauma Res $2016 ; 5(4): 1-5$.

6. Guven Y, Zorlu S, Cankaya AB, Aktoren O, Gencay K. A Complex Facial Trauma Case with Multiple Mandibular Fractures and Dentoalveolar Injuries. Case Rep Dent 2015; 2015:1-6.

7. de Alencar MGM, Rebelo HL, Júnior EZS, Junior MAB, Junior MDM. Tratamento de fratura complexa de mandíbula por abordagem transcervical: Relato de caso. Rev Cir Traumatol Buco-maxilo-fac 2015; 15(4):43-8.

8. Belli E, Liberatore G, Elidon M, Orabona GD'A, Piombino P, Maglitto F, et al. Surgical evolution in the treatment of mandibular condyle fractures. Bmc Surgery 2015; 15(1):2-6.

9. Boffano P, Kommers SC, Karagozoglu KH, Gallesio C, Forouzanfar T. Mandibular trauma: a two-centre study. Int J Oral Maxillofac Surg 2015; 44(8):998-1004.

10. Conci RA, Tomazi FHJ, Kalaoun R, Fritscher GG, de Oliveira GR, Heitz C. Modified submandibular access for open reduction and internal rigid fixation in condylar fractures. $\mathrm{J}$ Craniofac Surg 2015; 26(1):232-4.

11. Bouchard C, Mansouri M. Open Reduction with Internal Fixation of Mandibular Angle Fractures: A Retrospective Study. J Can Dent Assoc 2017; 83(3):1-6.

12. Munante-Cardenas JL, Facchina Nunes PH, Passeri LA. Etiology, treatment, and complications of mandibular fractures. J Craniofac Surg 2015; 26(3):611-5.
13. Afrooz PN, Bykouski MR, James IB, Daniali LN, Clavijo-Alvarez JA. The epidemiology od mandibular fractures in the United States, part 1: review of 13,142 cases from the US National Trauma Data Bank. J Oral Maxillofac Surg 2015; 73(12):2361-6.

14. Dantas BPSS, Fialho PV, Fernandes ACF, Silva DT, Queiroz CS. Fratura complexa de mandíbula: relato de caso. APCD 2017; 38(3):43-8.

15. Gomes ACA, Silva EDO, Carvalho R, Gomes DO, Feitosa DS, Maia SMH. Tratamento das fraturas mandibulares: relato de caso clínico. Rev Cir Trauma Buco-Maxilo-Fac 2001; $1(2): 31-8$

16. De Paula DM, Melo MNB, Souza SR, Dantas RMX, Dultra JA. Tratamento de pseudoartrose mandibular relato de caso. Rev Odontol Araçatuba 2017; 38(1):41-5.

\section{Endereço para correspondência:}

Rafael Drummond Rodrigues

Rua Manoel Gomes Ferreira, Conjunto Guilherme Marback, bloco 23, apartamento 301, setor 1, Imbuí 41706-060, Salvador, Bahia, Brasil

Telefone: (71) 99161-1871

E-mail:rafael_dr91@hotmail.com

Recebido: 18/09/18. Aceito: 26/10/18. 